${ }^{1}$ Centro de Medicina Reproductiva y Desarrollo Integral del Adolescente (CEMERA), Facultad de Medicina, Universidad de Chile. 2 Instituto Milenio para la Investigación en Depresión y Personalidad (MIDAP), Santiago, Chile.

${ }^{3}$ Departamento de Psicología, Facultad de Ciencias Sociales, Universidad de Chile.

${ }^{4}$ Escuela de Terapia Ocupacional, Facultad de Medicina, Universidad de Chile.

${ }^{5}$ Escuela de Salud Pública,

Facultad de Medicina, Universidad de Chile. ${ }^{6}$ Departamento de Cultura, Arte y Desarrollo Humano, Centro Universitario del Sur, Universidad de Guadalajara, Jalisco, México.

${ }^{7}$ Centro de Estudios Avanzados, Universidad de Playa Ancha, Valparaíso, Chile.

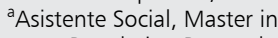
Population Research.

bEstadístico, Magíster en Bioestadística. 'Psicóloga Clínica.

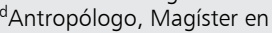
Psicología Clínica. essicólogo.

fPhD.

Fuente de financiamiento: Programas de Investigación Domeyko, Vicerrectoría de Investigación y Desarrollo, Universidad de Chile.

Recibido el 9 de junio de 2015, aceptado el 17 de diciembre de 2015.

Correspondencia a: Electra González A. Centro de Medicina Reproductiva y Desarrollo Integral del Adolescente (CEMERA). Facultad de Medicina, Universidad de Chile.

Profesor Alberto Zañartu 1030 Independencia, Santiago, Chile. Fax: 56-2-7356512. evgonzalez@med.uchile.cl

\title{
Diferencias de género en la calidad de vida relacionada con la salud en adolescentes escolarizados chilenos
}

\author{
ELECTRA GONZÁLEZ A. ${ }^{1, a}$, VANIA MARTÍNEZ N.$^{1,2}$, \\ TEMISTOCLES MOLINA G. ${ }^{1, \mathrm{~b}}$, MYRIAM GEORGE L., ${ }^{3, \mathrm{c}}$, \\ RODRIGO SEPÚLVEDA P., ${ }^{4, \mathrm{~d}, \mathrm{f}}$, RAMIRO MOLINA C. ${ }^{5}$, \\ CARLOS HIDALGO-RASMUSSEN $6,7, \mathrm{f}$
}

\section{Gender differences in health-related quality of life of Chilean adolescent students}

\begin{abstract}
Background: Health-related quality of life (HRQOL) refers to individuals perception of their subjective well-being, considering various aspects of their life and the impact on their health. Aim: To analyze gender differences in the HRQOL of adolescent students in Chile, by age, type of school attended, and area of residence. Material and Methods: Analytical cross-sectional study conducted in a population of $5^{\text {th }}$ and $12^{\text {th }}$ grade students attending municipal, subsidized and private schools in 11 regions of the country. HRQOL was assessed with the KIDSCREEN-52, an instrument that has been previously adapted and validated in Chile. The database obtained from that adaption and validation process was analyzed. Results: In total, 7,910 students (median age 14 years, 53\% female) completed the questionnaire. Compared to males, females had lower HRQOL scores in most of the KIDSCREEN-52 dimensions. However, males were more likely to have lower scores in the "Peers and Social Support" and "School Environment" dimensions. These differences remained valid when the sample was stratified by age, type of school, and area of residence were analyzed. Conclusions: This study supports the existence of inequalities in the self-perceived HRQOL of Chilean adolescent students. The existing differences are not only related to gender but are also evident when stratifying by type of school attended.
\end{abstract}

(Rev Med Chile 2016; 144: 298-306)

Key words: Adolescent; Quality of Life; Social Environment.
L a calidad de vida relacionada con la salud (CVRS) corresponde a la percepción que tienen las personas de su nivel de bienestar subjetivo, considerando diversos aspectos de su vida y su impacto en su estado de salud ${ }^{1}$.

Los estudios en CVRS inicialmente fueron realizados en población que presentaba patologías tanto agudas como crónicas y abarcan prácticamente todas las áreas de las especialidades en medicina $^{2,3}$. Posteriormente el interés se concentró en estudiar la CVRS en población general y luego en la población de niños y adolescentes ${ }^{4-6}$. Actualmente se han incrementado los estudios en CVRS en niños y adolescentes escolarizados tanto a nivel internacional ${ }^{7-11}$ como nacional ${ }^{12-14}$.

La Organización Panamericana de la Salud (OPS) promueve activamente la incorporación de la perspectiva de género a la salud pública para el diseño y la ejecución de políticas, programas y proyectos que abogan por la equidad de género siendo la promoción de la equidad en salud su mandato fundamental ${ }^{15}$. Desde esta perspectiva, la 
atención a las necesidades particulares de mujeres y hombres es clave, y en especial, en la población adolescente. Se reconocen sus diferencias y peculiaridades biológicas, así como su interacción con los factores sociales de género que suponen identidades, roles, responsabilidades y poderes de género diferentes, que se reflejan en los estados de salud de ambos sexos ${ }^{15}$. Las desigualdades de género en salud han sido estudiadas prioritariamente en población adulta, los estudios muestran que las mujeres declaran peor salud percibida que los varones, más síntomas emocionales o psicológicos y más dolor, problemas somáticos, depresión, así como la mayor utilización de los servicios de salud $^{16,17}$.

Los estudios que han abordado las diferencias de género en salud en niños, niñas y adolescentes coinciden en que a partir de la adolescencia las niñas auto perciben peor salud que los niños, a pesar que, en la mayoría, niñas, niños y adolescentes se consideran a sí mismos como saludables ${ }^{18,19}$. También indican que a mayor edad ambos sexos perciben peor su salud. Sin embargo, no todos los resultados son coincidentes, lo cual puede estar afectado por aspectos metodológicos como: las características de la muestra, los rangos de edades seleccionados, el contexto social de los estudios o los instrumentos de medición utilizados ${ }^{20,21}$.

Las diferencias biológicas podrían determinar diferente vulnerabilidad y susceptibilidad a enfermar según el sexo. Se ha descrito que las niñas son más propensas a declarar síntomas psicosomáticos o depresivos ${ }^{22}$, y problemas crónicos de salud ${ }^{23}$, asociado a una peor percepción de su salud ${ }^{24}$. También se ha encontrado diferencias en la forma en que los cambios puberales (físico, psicológicos) afectan la salud percibida, influyendo en forma negativa en las niñas ${ }^{25}$.

Los estudios en CVRS en niños y adolescentes realizados en varios países europeos ${ }^{8,20,26}$ encontraron diferencias por edad, esto es, que los niños y las niñas, poseen una mejor percepción de su CVRS cuando se compara con los y las adolescentes y por género, las niñas indicaron peores puntuaciones que los varones. Estas mismas diferencias fueron encontradas en estudios realizados en países latinoamericanos ${ }^{27,28}$. Esta peor percepción que presentan los adolescentes comparados con los niños puede relacionarse con la complejidad propia de esta etapa del desarrollo, en la cual, los individuos experimentan una creciente discrepancia entre su desarrollo físico e intelectual y psicológico por un lado y las posibilidades de independencia restringidas comparadas con los adultos, sobre todo cuando permanecen largo tiempo en el sistema escolar ${ }^{29}$.

Por otro lado, las diferencias de género, que se establecen a edades muy tempranas, pueden determinar o modificar la percepción que tienen los niños y niñas de su propia salud y calidad de vida ${ }^{20,30}$. Un momento clave de la influencia del medio en el desarrollo de los roles sociales y culturales ocurre en la infancia, en particular, en la etapa escolar. Es en el ambiente escolar donde se refuerzan las diferencias de género, a las niñas normalmente se les solicita tareas que involucran cuidados y a los niños se les piden tareas que involucran el uso de la fuerza ${ }^{31-32}$.

Finalmente, la evidencia demuestra que cuando el nivel socioeconómico decrece, la auto percepción de calidad de vida también decrece ${ }^{33}$, aunque algunos estudios no logran demostrar diferencias de género según nivel socioeconómico ${ }^{30}$.

En Chile es necesario evaluar la CVRS en adolescentes desde una perspectiva de género a nivel nacional para la formulación de políticas públicas e instalación de servicios y redes de atención en salud, por una parte, como formulación de políticas en el ámbito educativo que permitan modificar tempranamente actitudes, valores y conductas que perpetúan las desigualdades de género, en general, como conductas que perpetúan las desigualdades en salud.

El objetivo de este estudio fue analizar las diferencias de género en la percepción de CVRS en adolescentes escolarizados chilenos por edad, colegio según administración y zona del país.

\section{Material y Método}

\section{Diseño}

Estudio transversal y analítico, la población de estudio estuvo constituida por estudiantes que cursaban desde quinto básico hasta cuarto medio. La información se obtuvo de una base de datos utilizada para la adaptación y validación de KIDSCREEN-52. El trabajo de campo se realizó entre julio y octubre de 2009. El cálculo del tamaño muestral con significación nacional se basó en la información de la población chilena escolar con edades de 10 hasta 18 años ${ }^{13}$. El tipo de muestreo, selección de alumnos, como se recolectó la in- 
formación y diseño de muestras comparativas se describieron en una publicación anterior ${ }^{14}$.

\section{Variables de estudio}

Variables dependientes.

Calidad de Vida Relacionada con la Salud.

Las variables dependientes corresponden a las 10 dimensiones del instrumento KIDSCREEN-52 ${ }^{34}$ utilizadas para la versión chilena ${ }^{14}$ con un total de 52 ítems, cuyo número se señala entre paréntesis: Bienestar físico (5), Bienestar psicológico(6), Estado de ánimo y emociones (7), Auto percepción (5), Autonomía (5), Relación con los padres y vida familiar (6), Recursos económicos (3), Amigos y apoyo social (6), Entorno escolar (6) y Aceptación social (bullying) (3). Las opciones de respuesta para los 52 ítems están en escala de Likert, con 5 puntos (desde nunca $=1$, hasta siempre $=5) . \mathrm{El}$ recordatorio fue la última semana.

En Europa las dimensiones tienen validez y consistencia internas adecuadas ${ }^{34}$, en Chile se verificó su validez mediante análisis factorial confirmatorio $^{14}$ y fiabilidad, la escala obtuvo alfa de Cronbach para las dimensiones mayores a 0,70 , a excepción de Auto percepción, con 0,62 ${ }^{14}$.

Los ítemes para cada dimensión se sumaron, en función de estos puntajes, se utilizó el modelo teórico de crédito parcial (PCM) que pertenece a la familia de los modelos Rasch, para calcular los puntajes Rasch para cada dimensión ${ }^{35}$. El valor de los estadísticos INFIT y OUTFIT tanto para los ítems como para las personas, reflejaron un buen ajuste de los datos al modelo. Estas puntuaciones fueron posteriormente trasformados a valores $\mathrm{T}$, correspondientes a una media de 50 y una desviación estándar de 10, para una mayor facilidad de uso. Se utilizó el programa WINSTEP para realizar el análisis de los Puntajes Rasch ${ }^{35}$.

Puntuaciones menores significan percepción de baja CVRS. Las dimensiones se dicotomizaron usando una DE como punto de corte de acuerdo a lo sugerido en estudios anteriores ${ }^{36,37}$. Para cada dimensión se codificó la puntuación T $(0=$ CVRS alto, $1=$ CVRS bajo).

\section{Variables independientes}

Nivel individual.

Sexo (dummy mujer $=1$ ).

Nivel agregado.

Nivel socioeconómico del colegio: (dummy nivel socioeconómico medio $=1$, dummy nivel socioeconómico alto $=1$ ).

Unidades de nivel agregado

Colegios participantes: colegios del área urbana seleccionados aleatoriamente para el estudio original en 11 regiones del país ${ }^{14}$.

Variables de estratificación

Edad: en rangos de: 10-12 años, 13-15 años y 16-18 años.

Tipo de colegio según administración del establecimiento: municipalizado, particular subvencionado, particular pagado.

Zonas: Zona Norte (Arica y Parinacota, Iquique, Antofagasta, Copiapó, Coquimbo), Zona Central (Valparaíso y Región Metropolitana) y Zona Sur (Maule, Bío-Bío, De los Lagos, Magallanes).

\section{Análisis de datos}

Considerando que los residuos de la CVRS en las 10 dimensiones no tuvieron una distribución normal y que las observaciones a nivel individual se encontraban anidadas dentro del tipo de colegio, para analizar la relación entre la CVRS en adolescentes escolarizados chilenos y género en las 10 dimensiones del KIDSCREEN-52 se utilizó regresión logística multinivel ${ }^{38,39}$ para cada una de las diez dimensiones.

\section{Modelo}

Variable dependiente (Dimensión): Variables independientes (Sexo, NSE del colegio).

Unidad de nivel agregado (colegios participantes).

Para tener una primera aproximación de la relación entre estas variables antes se realizó un análisis sin estratificar. Luego cada modelo fue estratificado por Grupo de edad, Tipo de colegio y Zona.

La bondad de ajuste se midió a través de CCI: Coeficiente de correlación intraclase; PVE: Porcentaje de varianza explicado; MRM: Mediana de razón de momios.

Se utilizó el software estadístico STATA 12.0 (StataCorp LP, Texas, USA).

A todos los participantes se les solicitó su asentimiento y el consentimiento de sus padres o tutores para participar en el estudio. El estudio fue aprobado por el Comité de Ética en Seres Humanos de la Facultad de Medicina de la Universidad de Chile. 


\section{Resultados}

Los resultados correspondieron a 7.910 adolescentes escolarizados de ambos sexos que contestaron y completaron el cuestionario. El 53\% fueron mujeres. La edad promedio a la encuesta fue de 14 años $(\mathrm{DE}=2,3)$, presentando las mujeres un promedio de 13,7 años $(\mathrm{DE}=2,3)$ y los hombres $14,3(\mathrm{DE}=2,3)$. Mediante la regresión logística multinivel vacía se pudo cuantificar por separado la varianza que podemos atribuir al nivel individual y al agregado. Cuando se consideró el modelo nulo, el CCI para las 10 dimensiones fluctúo entre 2,6\% (Auto percepción) y 9,4\% (Aceptación social (bullying)), indicando que entre estos porcentajes de la variabilidad observada de CVRS corresponde a la diferencia entre tipos de colegio. El porcentaje de varianza explicado (PVE) en el modelo final varió entre 11,1\% para "Entorno escolar" y 46,6\% para "Amigos y apoyo social" (Tabla 1).

$\mathrm{Al}$ ajustar el modelo multinivel sin estratificar las mujeres presentaron mayor riesgo de presentar puntajes más bajos en 7 dimensiones de CVRS (Bienestar físico, Bienestar psicológico, Estado de ánimo y emociones, Auto percepción, Autonomía, Relación con los padres y vida familiar y Recursos económicos) en relación a los hombres (RM: 1,21-2,61); en las dimensiones "Amigos y apoyo social" y "Entorno escolar" las mujeres presenta- ron puntajes de riesgo más bajos que los hombres. La dimensión "Aceptación social” (bullying) no presentó diferencia (Tabla 1).

$\mathrm{Al}$ estratificar por grupo de edad las mujeres presentaron mayor riesgo de presentar puntajes más bajos en 6 dimensiones de CVRS (Bienestar físico, Bienestar psicológico, Estado de ánimo y emociones, Auto percepción, Autonomía, Relación con los padres y vida familiar) en relación a los hombres en los 3 grupos de edad. En el grupo de 10 a 12 años en la dimensión "Bienestar físico" las mujeres presentaron 2,15 veces más riesgo que los hombres de presentar puntaje bajo, siendo este puntaje el mayor respecto del resto de las dimensiones. En el grupo de 13 a 15 años en la dimensión "Estado de ánimo y emociones" las mujeres presentaron 2,44 veces más riesgo que los hombres de presentar puntaje bajo siendo este el mayor respecto del resto de las dimensiones. En el grupo de 16 a 18 años en la dimensión "Bienestar físico" las mujeres presentaron 3,59 veces más riesgo que los hombres de presentar puntaje bajo siendo este puntaje el mayor respecto del resto de las dimensiones (Tabla 2).

$\mathrm{Al}$ estratificar por tipo de colegio las mujeres presentaron mayor riesgo de presentar puntajes más bajos en 6 dimensiones de CVRS (Bienestar físico, Bienestar psicológico, Estado de ánimo y emociones, Auto percepción, Autonomía, Rela-

Tabla 1. Modelo nulo, modelo ajustado de regresión logística multinivel entre dimensiones de la CVRS y sexo ajustado por NSE del colegio y variable agregada Tipo de colegio

\begin{tabular}{|lcccccc|}
\hline Dimensión & \multicolumn{2}{c}{ Modelo nulo } & \multicolumn{4}{c|}{ Modelo ajustado } \\
& CCI & MRM & CCI & PVE & MRM & RM (IC 95\%) \\
\hline Bienestar físico & 0,068 & 1,60 & 0,051 & 27,3 & 1,49 & $2,61(2,25-3,03)$ \\
\hline Bienestar psicológico & 0,039 & 1,42 & 0,035 & 12,9 & 1,39 & $1,60(1,39-1,84)$ \\
\hline Estado de ánimo y emociones & 0,047 & 1,47 & 0,029 & 38,0 & 1,35 & $1,97(1,71-2,27)$ \\
\hline Autopercepción & 0,026 & 1,32 & 0,019 & 24,1 & 1,28 & $2,07(1,80-2,39)$ \\
\hline Autonomía & 0,046 & 1,46 & 0,052 & $-11,4$ & 1,50 & $1,61(1,40-1,86)$ \\
\hline Relación con los padres y vida familiar & 0,058 & 1,54 & 0,038 & 35,1 & 1,41 & $1,69(1,47-1,93)$ \\
\hline Recursos económicos & 0,069 & 1,60 & 0,039 & 45,8 & 1,42 & $1,21(1,06-1,37)$ \\
\hline Amigos y apoyo social & 0,037 & 1,41 & 0,020 & 46,6 & 1,28 & $0,83(0,72-0,95)$ \\
\hline Entorno escolar & 0,063 & 1,57 & 0,057 & 11,06 & 1,53 & $0,85(0,74-0,97)$ \\
\hline Aceptación social (bullying) & 0,094 & 1,75 & 0,080 & 16,8 & 1,66 & $0,94(0,81-1,08)$ \\
\hline
\end{tabular}

RM: Razón de momios (OR, odds ratio) Sexo (dummy mujer = 1); IC: Intervalo de confianza; CCl: Coficiente de correlación intraclase; PVE: Porcentaje de varianza explicado; MRM: Mediana de razón de momios. CVRS: Calidad de Vida Relacionada con la Salud, - punto de corte corresponde a una DE de puntuación T ( 0 = CVRS mayor, 1 = CVRS menor). 
ción con los padres y vida familiar) en relación a los hombres en los 3 tipos de colegios. En el tipo de colegio municipal en la dimensión "Bienestar físico" las mujeres presentaron 2,75 veces más riesgo que los hombres de presentar puntaje bajo siendo este puntaje el mayor respecto del resto de las dimensiones. En el tipo de colegio subvencionado en la dimensión "Estado de ánimo y emociones" las mujeres presentaron 1,83 veces más riesgo que los hombres de presentar puntaje bajo siendo éste el mayor respecto del resto de las dimensiones. En el tipo de colegio particular en la dimensión "Auto percepción" las mujeres presentaron 2,53 veces más riesgo que los hombres de tener puntaje bajo respecto del resto de las dimensiones, siendo éste el mayor respecto de las otras dimensiones (Tabla 3).

Tabla 2. Regresión logística multinivel entre dimensiones de la CVRS y sexo ajustado por NSE del colegio y variable agregada Tipo de colegio, según grupo etario en puntajes Rasch

\begin{tabular}{|c|c|c|c|c|c|c|}
\hline \multirow[t]{2}{*}{ Dimensiones } & \multicolumn{2}{|c|}{10 a 12 años } & \multicolumn{2}{|c|}{13 a 15 años } & \multicolumn{2}{|c|}{16 a 18 años } \\
\hline & $\mathbf{R M}$ & I C 95\% & $\mathbf{R M}$ & I C 95\% & $\mathbf{R M}$ & I C 95\% \\
\hline Bienestar físico & 2,15 & $1,58-2,95$ & 2,36 & $1,85-2,99$ & 3,59 & $2,89-4,45$ \\
\hline Bienestar psicológico & 1,67 & $1,23-2,25$ & 1,79 & $1,44-2,23$ & 1,56 & $1,25-1,95$ \\
\hline Estado de ánimo y emociones & 1,49 & $1,13-1,97$ & 2,44 & $1,95-3,05$ & 2,11 & $1,66-2,68$ \\
\hline Autopercepción & 1,66 & $1,25-2,20$ & 2,04 & $1,63-2,54$ & 2,71 & $2,16-3,42$ \\
\hline Autonomía & 1,70 & $1,28-2,25$ & 1,53 & $1,23-1,91$ & 1,66 & $1,33-2,08$ \\
\hline Relación con los padres y vida familiar & 1,77 & $1,32-2,38$ & 2,07 & $1,68-2,54$ & 1,50 & $1,21-1,87$ \\
\hline Recursos económicos & 0,96 & $0,77-1,20$ & 1,32 & $1,08-1,63$ & 1,34 & $1,06-1,69$ \\
\hline Amigos y apoyo social & 0,90 & $0,71-1,14$ & 0,56 & $0,44-0,70$ & 1,10 & $0,88-1,39$ \\
\hline Entorno escolar & 0,80 & $0,60-1,06$ & 0,86 & $0,69-1,08$ & 0,96 & $0,77-1,21$ \\
\hline Aceptación social & 0,93 & $0,75-1,15$ & 0,89 & $0,71-1,12$ & 0,77 & $0,57-1,04$ \\
\hline
\end{tabular}

RM: Razón de momios (OR, odds ratio) Sexo (dummy mujer $=1$ ); IC: Intervalo de confianza; Puntaje T (media de 50 y Ds. de 10), utilizando los puntajes Rasch chilenos.

Tabla 3. Regresión logística multinivel entre dimensiones de la CVRS y sexo ajustado por NSE del colegio y variable agregada Tipo de colegio, según tipo de establecimiento en puntajes Rasch

\begin{tabular}{|lcccccc|}
\hline Dimensiones & \multicolumn{2}{c}{$\begin{array}{c}\text { Municipalizado } \\
\text { RM }\end{array}$} & I C 95\% & \multicolumn{2}{c}{$\begin{array}{c}\text { Subvencionado } \\
\text { RM }\end{array}$} & \multicolumn{2}{c|}{$\begin{array}{c}\text { Particular } \\
\text { I C 95\% }\end{array}$} & RM & I C 95\% \\
\hline Bienestar físico & 2,75 & $2,15-3,52$ & - & - & 2,30 & $1,74-3,04$ \\
\hline Bienestar psicológico & 1,88 & $1,48-2,40$ & 1,73 & $1,36-2,19$ & 1,22 & $0,94-1,58$ \\
\hline Estado de ánimo y emociones & 2,33 & $1,82-3,00$ & 1,83 & $1,47-2,29$ & 1,77 & $1,35-2,32$ \\
\hline Autopercepción & 1,99 & $1,55-2,59$ & 1,77 & $1,42-2,22$ & 2,53 & $1,95-3,27$ \\
\hline Autonomía & 1,42 & $1,09-1,84$ & 1,48 & $1,18-1,86$ & 1,98 & $1,55-2,54$ \\
\hline Relación con los padres y vida familiar & 1,66 & $1,32-2,08$ & 1,77 & $1,42-2,21$ & 1,63 & $1,25-2,12$ \\
\hline Recursos económicos & 1,16 & $0,95-1,43$ & 1,32 & $1,08-1,62$ & 1,05 & $0,80-1,37$ \\
\hline Amigos y apoyo social & 0,85 & $0,68-1,07$ & 0,76 & $0,61-0,95$ & 0,85 & $0,65-1,11$ \\
\hline Entorno escolar & 0,80 & $0,63-1,01$ & 0,83 & $0,66-1,05$ & 0,91 & $0,70-1,17$ \\
\hline Aceptación social & 1,16 & $0,92-1,46$ & 0,74 & $0,58-0,93$ & 0,97 & $0,73-1,29$ \\
\hline
\end{tabular}

RM: Razón de momios (OR, odds ratio) Sexo (dummy mujer = 1); IC: Intervalo de confianza; Puntaje T (media de 50 y Ds. de 10), utilizando los puntajes Rasch chilenos. 
Tabla 4. Regresión logística multinivel entre dimensiones de la CVRS y sexo ajustado por NSE del colegio y variable agregada Tipo de colegio, según zona del país en puntajes Rasch

\begin{tabular}{|lcccccc|}
\hline Dimensiones & \multicolumn{2}{c}{ Zona Norte } & \multicolumn{2}{c}{ Zona Centro } & \multicolumn{2}{c|}{ Zona Sur } \\
& RM & I C 95\% & RM & I C 95\% & RM & I C 95\% \\
\hline Bienestar físico & 2,54 & $1,90-3,39$ & 2,88 & $2,34-3,54$ & 1,99 & $1,41-2,80$ \\
\hline Bienestar psicológico & 2,0 & $1,51-2,67$ & 1,50 & $1,24-1,80$ & 1,43 & $1,02-2,0$ \\
\hline Estado de ánimo y emociones & 2,41 & $1,79-3,27$ & 2,01 & $1,67-2,42$ & 1,47 & $1,05-2,07$ \\
\hline Autopercepción & 2,39 & $1,78-3,22$ & 2,10 & $1,75-2,52$ & 1,70 & $1,23-2,36$ \\
\hline Autonomía & 1,64 & $1,23-2,19$ & 1,65 & $1,37-2,0$ & 1,42 & $1,04-1,92$ \\
\hline Relación con los padres y vida familiar & 1,77 & $1,35-2,33$ & 1,68 & $1,41-2,01$ & 1,60 & $1,15-2,22$ \\
\hline Recursos económicos & 1,20 & $0,92-1,58$ & 1,30 & $1,10-1,54$ & 0,99 & $0,74-1,32$ \\
\hline Amigos y apoyo social & 0,69 & $0,53-0,90$ & 0,87 & $0,72-1,04$ & 0,91 & $0,68-1,22$ \\
\hline Entorno escolar & 0,92 & $0,69-1,22$ & 0,85 & $0,71-1,03$ & 0,72 & $0,52-0,99$ \\
\hline Aceptación social & 1,0 & $0,75-1,33$ & 0,98 & $0,81-1,18$ & 0,75 & $0,54-1,04$ \\
\hline
\end{tabular}

RM: Razón de momios (OR, odds ratio) Sexo (dummy mujer =1); IC: Intervalo de confianza; Puntaje T (media de 50 y Ds. de 10), utilizando los puntajes Rasch chilenos.

Al estratificar por zona las mujeres presentaron mayor riesgo de presentar puntajes más bajos en 6 dimensiones de CVRS (Bienestar físico, Bienestar psicológico, Estado de ánimo y emociones, Autopercepción, Autonomía, Relación con los padres y vida familiar) en relación a los hombres en las 3 zonas del país. En la zona Norte en la dimensión "Bienestar físico" las mujeres presentaron 2,54 veces más riesgo que los hombres de presentar puntaje bajo. En la zona Centro en la dimensión "Bienestar físico" las mujeres presentaron 2,88 veces más riesgo que los hombres de presentar puntaje bajo. En la zona Sur en esta dimensión las mujeres presentaron 1,99 veces más riesgo que los hombres de presentar puntaje bajo (Tabla 4 ).

\section{Discusión}

El presente estudio mostró diferencias de género en la CVRS percibida por los adolescentes escolarizados chilenos, lo que es concordante con lo reportado por otros estudios tanto europeos ${ }^{18-21}$ como latinoamericanos ${ }^{22-24}$.

Las mujeres presentaron puntajes más bajos en su auto percepción de CVRS en las dimensiones "Bienestar físico", "Bienestar psicológico", "Estado de ánimo", "Auto percepción”, "Autonomía", "Relación con los padres y vida familiar" en relación a los hombres. Coincidente con otros estudios, en las dimensiones "Amigos y apoyo social" y "Entorno escolar", las mujeres presentaron puntajes más altos que los hombres ${ }^{18-24}$.

Los varones presentaron mayor riesgo de puntajes bajos en las dimensiones "Amigos y apoyo social" y "Entorno escolar", comparados con las mujeres. Esta peor percepción sobre el ambiente escolar seguramente se relaciona con la mayor complejidad que caracteriza esta etapa del desarrollo para los varones en nuestro sistema escolar por lo que es importante considerar cual es el rol que está jugando la escuela y como ésta contribuye a esta complejidad. No se encontró estudios nacionales que permitan explicar por qué los varones chilenos presentan un mayor riesgo en estas dimensiones. En la dimensión "Amigos y apoyo social" se valora positivamente el apoyo mutuo, la conversación íntima y el nivel de confianza con los pares. Aspectos que pudieran ser más valorados por el género femenino que por el masculino. Sería un importante punto de intervención preventiva, ya que los hombres frente a los problemas interpersonales pueden tender a utilizar mecanismos más evasivos, incluyendo el abuso de alcohol y otras sustancias. Por otro lado, en estudios internacionales se ha encontrado que los adolescentes hombres presentan más sintomatología externalizante que se asocia 
a trastorno oposicionista desafiante, síndrome de déficit atencional y trastorno de conducta ${ }^{40}$. Esto podría explicar una peor percepción de la dimensión "Entorno escolar", en la que se incluyen preguntas de nivel de atención y relación con los profesores entre otras. Ofrecer atención en salud mental a adolescentes con esta problemática y a la vez, fortalecer las habilidades de los profesores para su mejor manejo serían útiles para una mejor percepción de esta dimensión en los adolescentes hombres.

Si bien hombres y mujeres reportaron puntajes más bajos en su percepción de CVRS a medida que aumenta la edad, llama la atención que en las edades de 10 a 12 años y 16 a 18 años las mujeres presentaron más riesgo de puntaje bajo en la dimensión "Bienestar físico", mientras que en el grupo de 13 a 15 años fue la dimensión "Estado de ánimo y emociones". Esto constituye una señal de alerta para las autoridades tanto educacionales como de salud. Las adolescentes mujeres tienen más sobrepeso y obesidad y tienen menos actividad física que los adolescentes hombres ${ }^{41}$. Además, antes de la pubertad la prevalencia de depresión es similar en ambos géneros, después de la pubertad las mujeres tienen más riesgo de tener depresión ${ }^{42}$. Sería interesante en nuevos estudios incorporar la relación entre depresión, sobrepeso u obesidad y CVRS.

El presente estudio mostró que las diferencias de género en la percepción de CVRS de los escolares chilenos permanecieron al analizar la variable económica (medida indirectamente a través del tipo de administración del colegio) y la zona del país.

Finalmente la percepción del Estado de Ánimo y Emociones de los escolares chilenos es preocupante, ya que alcanza puntajes más bajos que lo reportado en estudios europeos ${ }^{7-9}$, especialmente en mujeres de colegios municipalizados, esto también constituye una señal de alerta para las autoridades, debido a que puede estar relacionado a riesgo de depresión o depresión establecida, lo que a su vez puede aumentar el riesgo de suicidio. Por lo tanto, es indispensable conocer y analizar más en profundidad esta información y monitorizarla.

Los resultados de este estudio mostraron que las mujeres adolescentes escolarizadas en Chile perciben una peor CVRS cuando se compara con los varones, independiente de su edad, tipo de colegio y zona del país.
Dado que ya se cuenta con la adaptación transcultural y la validación en nuestro país del instrumento KIDSCREEN-52, al incluir la perspectiva de género, este estudio, puede contribuir de manera innovadora al diseño y mejoramiento de las políticas educacionales y de salud, a nivel nacional y regional, de niños y adolescentes. Si se incorpora la visión de los propios escolares, tomando en cuenta sus diferencias de género, se promoverá las buenas prácticas que mejoraran su CVRS.

Limitaciones del estudio: Es un estudio transversal lo que impide conocer la relación de causalidad. La muestra no incluyó colegios de zonas rurales lo cual dificulta la generalización a la población rural escolarizada de Chile que corresponde a $14,3 \%$ del total de escolares de Chile ${ }^{43}$. El nivel socioeconómico no fue medido directamente lo que puede haber afectado los resultados en relación a esta variable.

Agradecimientos: Los autores agradecen a todos los estudiantes y profesores quienes generosamente dieron su tiempo para participar en el estudio.

\section{Referencias}

1. WHO. Quality Life Group. The World Health Organization Quality Life Assessment instrument (WHOQOL): position paper from the World Health Organization. Soc Sci Med 1995; 41 (10): 1403-9.

2. Willke RJ, Burke LB, Erickson P. Measuring treatment impact: a review of patient-reported outcomes and other efficacy and points in approved product levels. Control Clin Trials 2004; 25 (6): 535-52.

3. Miranda MJ, Domínguez E, Arroyo FJ, Méndez P, González J. Calidad de vida relacionada con la salud en la diabetes mellitus tipo 1. An Pediatr (Barc) 2012; 77 (5): 329-33.

4. Solans M, Pane S, Estrada MD. Health-related quality of life measurement in children and adolescents: A systematic review of generic and disease-specific instruments. Value Health 2008; 11 (4): 742-64.

5. Badia X, Benavides A, Rajmil L. Instruments for measuring health-related quality of life in children and adolescents with asthma. An Esp Pediatr 2001; 54 (3): 213-21.

6. Avendaño MJ, Barra E. Auto-eficacia, Apoyo Social y Calidad de Vida en Adolescentes con Enfermedades Crónicas. Terapia Psicológica 2008; 26 (2): 165-72. 
7. Ravens-Sieberer U, Gosch A, Rajmil L, Erhart M, Bruil J, Duer W, et al. KIDSCREEN Group E. KIDSCREEN-52 quality-of-life measure for children and adolescents. Expert Rev Pharmacoecon Outcomes Res 2005; 5 (3): 353-64.

8. Aymerich M, Berra S, Guillamon I, Herdman M, Alonso J, Ravens-Sieberer U, et al. Desarrollo de la versión en español del KIDSCREEN, un cuestionario de calidad de vida para la población infantil y adolescente. Gac Sanit 2005; 19 (2): 93-102.

9. Ravens-Sieberer U, Gosch A, Rajmil L, Erhart M, Bruil J, Power M, et al. KIDSCREEN Group. THE KIDSCREEN-52 Quality of life measure for children and adolescents: psychometric results from a cross-cultural survey in 13 European countries. Value Health 2008; 11 (4): 645-58.

10. Quintero C, Lugo L, García H, Sánchez A. Validación del cuestionario KIDSCREEN-27 de calidad de vida relacionada con la salud en niños y adolescentes de Medellín, Colombia. Rev Colomb Psiquiat 2011; 40: 470-87.

11. Gerharz E, Eiser C, Woodhouse C. Current approaches to assessing the quality of life in children and adolescents. BJU International 2003; 91 Suppl. 2: 150-4.

12. Urzúa A, Cortés E, Prieto L, Vega S, Tapia K. Autoreporte de la calidad de vida en niños y adolescentes escolarizados. Rev Chil Pediatr 2009; 80 (3): 238-44.

13. Hidalgo-Rasmussen C, Molina T, Molina R, Sepúlveda R, Martínez V, Montaño R, et al. Bullying y calidad de vida relacionada con la salud en adolescentes escolares. Rev Med Chile 2015; 143 (6): 716-23.

14. Sepúlveda R, Molina T, Molina R, Martínez V, González $\mathrm{E}$, George $\mathrm{M}$, et al. Adaptación y validación de un instrumento de calidad de vida relacionada con la salud en adolescentes chilenos. Rev Med Chile 2013; 141: 128392.

15. Sabo D. Comprender la salud de los hombres. Un enfoque relacional y sensible al género. OPS. Harvard Center and Development Studies, 2000. Publicación Ocasional No 4.

16. Benyamin $Y$, Leventhal H. Gender differences in processing information for making self-assessments of health. Psy Med 2000; 62: 354-64.

17. Fernández E, Schiaffino A, Rajmil L,Badia X, Segura A. Gender inequalities in health and health care a services use in Catalonia (Spain). J Epidemiol Community Health $1999 ; 53: 218-22$.

18. Cavallo F, Zambon A, Borraccino A, Raven-Sieberer U, Torsheim T, Lemma P, \& the HBSC Positive Health Group. Girls growing through adolescence have a higher risk of poor health. Qual Life Res 2006; 15: 1577-85.
19. Serra-Sutton V, Rajmil L, Aymerich M. Estrada MD. Desigualtats de genero en la percepció de la salut durant l'adolescencia. Annals de Medicina 2004; 87: 25-9.

20. Bisegger, Letta B, Von Rueden U. The European KIDSCREEN Group et al. Health-related quality of life: gender differences in Childhood and adolescence. Soz-Praventivmed 2005; 50: 281-91.

21. Collier J, MacKinlay D, Phillips D. Norm-values for the generic Children's quality of life measure. (GCQ) from a large school-based sample. Qual Life Res 2000; 9 (6): 617-23.

22. Svavarsdottir EK, Orlygsdottir B. Health-related quality of life in Iceland school children. Scand J Caring Sci 2006; 20: 209-15.

23. Piko BF. Self-perceived health among adolescents: the role of gender and psychological factors. Eur J Pediatr 2007; 166: 701-8.

24. Vélez R, López S, Rajmil L. Género y salud percibida en la infancia y la adolescencia en España. Gac Sanit 2009; 433-9.

25. Williams PG, Colder CR, Richard MH, Richards MH, Scalzo C. The role of self-assessed health in the relationship between gender and depressive symptoms among adolescents. J Pediatr Psychol 2002; 27: 509-17.

26. Tebe C, Berra S, Herdman M, Aymerich M, Alonso J, Rajmil L. Fiabilidad y validez de la versión española del KIDSCREEN-52 para población infantil y adolescente. Med Clin 2008; 130 (17): 650-4.

27. Berra S, Tebe C, Esandi ME, Carignano C. Reliability and validity of the KIDSCREEN-52 questionnaire to measure health related quality of life in the 8 to 18 yearold Argentinean population 2013; 111 (1): 29-35.

28. Hidalgo-Rasmussen C, Rajmil L, Montaño R. Adaptación transcultural del cuestionario KIDSCREEN para medir calidad de vida relacionada con la salud en población Mexicana de 8 a 18 años. Cien Saude Colet (Internet) 2014; 19 (7): 2215-24.

29. Moffits TE. Adolescence-limited and life-course-persistent antisocial behavior: a developmental taxonomy. Psychol Rev 1993; 100: 674-701.

30. Courtenay WH. Constructions of masculinity and their influence on men well-being: a theory of gender and health. Soc Sci Med 2000; 50: 1385-401.

31. Padilla MT, García S, Suárez M. Diferencias de género en el autoconcepto general y académico de estudiantes de $4^{\circ}$ de ESO. Revista de Educación 2010; 352: 495 515.

32. Gomes G, Soares A. Diferencias de género con relación al desempeño académico en estudiantes de nivel básico. Alternativas en Psicología 2013; 17 (28): 106-18.

33. Berra S, Bustingorry V, Henze C, Díaz P, Rajmil L, 
Butinof M. Adaptacion transcultural del cuestionario KIDSCREEN para medir calidad de vida relacionada con la salud en población Argentina de 8 a 18 años. Arch Argent Pediatric 2009; 107 (4): 307-14.

34. Ravens-Sieberer U, Gosch A, Rajmil L, Erhart M, Bruil J, Power M, et al. The KIDSCREEN-52 quality of life measure for children and adolescents: Psychometric results from a cross-cultural survey in 13 European countries. Value Heal 2008; 11 (4): 645-58.

35. Linacre J. 2003. A user guide to winsteps. Rasch model computer program. Chicago, IL: MESA edition.

36. Analitis F, Velderman MK, Ravens-Sieberer U, Detmar S, Erhart M, Herdman M, et al. Being bullied: associated factors in children and adolescents 8 to 18 years old in 11 European countries. Pediatrics [Internet]. 2009 Feb [Consultado el 18 de junio de 2014]; 123 (2): 569-77. Disponible en: http://www.ncbi.nlm.nih.gov/pubmed/19171624.

37. The Kidscreen Group Europe. The KIDSCREEN questionnaires: Quality of life questionnaires for children and adolescents. Lengerich. Pabst Science Publishers; 2006.

38. Multinivel and Longitudinal Modeling using Stata Sophia Rabe-Hesketh, Anders Skrondal (2012) 3ra ed.

39. Introducción al análisis multinivel. Cuadernos Metodológicos no 49. Héctor Cebolla Boado.

40. Carragher N, Teesson M, Sunderland M, Newton NC, Krueger RF, Conrod PJ, et al. The structure of adolescent psychopathology: a symptom-level analysis, Psycho Med 2015; 1: 1-14.

41. Azar AL, Franetovic G, Martínez M, Santis H. Determinantes individuales, sociales y ambientales del sobrepeso y la obesidad adolescente en Chile. Rev Med Chile 2015; 143 (5): 598-605.

42. Birmaher B, Ryan ND, Williamson DE, Brent DA, Kaufman J, Dahl RE, et al. Childhood and adolescent depression: a review of the past 10 years, Part I, J Am Acad Child Adolesc Psychiatry 1996; 35 (11): 1427-39.

43. Estadísticas sobre Escuela Rural, Ministerio de Educación, Dirección General de Educación, 2008. 